Background Subcutaneous Levetiracetam is increasingly used to control seizure activity in selected palliative patients. Despite this becoming a well-recognised approach quality evidence to support this remains sparse. This retrospective audit explores the use of subcutaneous Levetiracetam in palliative patients across the whole of the West Midlands, UK.

Methodology West Midland based Specialist Palliative Care Units $(n=14)$ and Hospital Teams $(n=17)$ were invited to participate in an electronic survey collecting anonymised retrospective data on patients in whom subcutaneous Levetiracetam had been used. Information gathered included; seizure aetiology and type, antiepileptic history, delivery of Levetiracetam, side effects and effectiveness.

Results Information generated from 31 cases demonstrated subcutaneous Levetiracetam use in a wide range of seizure aetiologies (space-occupying lesions (50\%), pre-existing epilepsy, cerebrovascular disease, seizures secondary to Creutzfledt-Jakob disease, leptomeningeal disease and Multiple Sclerosis). $48 \%$ patients had experienced seizure activity within the week prior to commencement on subcutaneous Levetiracetam and nearly all (93\%) were already using antiepileptic drugs. Levetiracetam was delivered most commonly via a McKinley T34ㄷ continuous subcutaneous infusion (84\%). The median dose of Levetiracetam on commencement was 1000 $\mathrm{mg}$ (range $250 \mathrm{mg}-3000 \mathrm{mg}$ ) and $12 \%$ of infusions were titrated over time due to seizure activity.

Levetiracetam was successfully mixed with Morphine, Midazolam, Metoclopramide and Dexamethasone. Concurrent Midazolam administration was used in $68 \%$ due to varying rationale. $81 \%$ reported no side effects attributable to Levetiracetam and $16 \%$ reported a local skin site reaction. No further seizures were documented in $70 \%$, and $65 \%$ subcutaneous Levetiracetam continued until death.

Conclusions This study outlines current practice within the West Midlands, adds to the relatively small evidence base, will help inform the composition of regional guidelines and provide a platform upon which to develop future research.

\section{STAFF PERCEPTIONS OF NON-PHARMACOLOGICAL INTERVENTIONS TO MANAGE DELIRIUM IN THE INPATIENT PALLIATIVE CARE SETTING}

Claire Conway, Juliet Spiller, Emma Carduff, Anne Finucane. University of Edinburgh

\subsection{6/bmjspcare-2018-ASPabstracts. 198}

Background and aim Delirium is a complex neuropsychiatric syndrome occurring in up to $88 \%$ of patients in palliative care inpatient settings in the weeks or hours preceding death. It is characterised by a disturbance in attention and awareness that develops over a short time and fluctuates in severity throughout the day. Management of a delirium episode includes the consideration of potentially treatable precipitating and aggravating factors followed by symptomatic treatment with drug therapy. A recent randomised clinical trial found no benefit of antipsychotic medication in the treatment of terminally ill patients, and called for an examination of the role of supportive interventions (Agar et al. 2017). The aim of the present study was to explore staff perspectives regarding supportive or non-pharmacological strategies to manage delirium in palliative care settings.

Methods Semi-structured interviews were conducted with nine members of the medical and nursing inpatient teams across two Scottish hospices. Interviews were transcribed verbatim and analysed thematically.

Results Most participants employed a mix of pharmacological and non-pharmacological approaches to treat delirium at the end of life. Non-pharmacological strategies included i) reorientation, ii) modifying environmental factors, iii) familiar surroundings, iv) enabling and retaining autonomy, v) distraction from delirium, and vi) tactile stimulation.

Inadequate staffing was viewed as a barrier to the use of non-pharmacological interventions; whereas involving families in delirium management was viewed as helpful.

Conclusion A variety of non-pharmacological interventions were identified which were both commonly used and perceived to be effective by staff members in two palliative care inpatient units. An intervention study is now required to examine the effectiveness of supportive interventions to reduce delirium symptoms and severity in terminally ill patients, and to minimise delirium related distress in both the patient and their families.

\section{PILOT STUDY: POINT PREVALENCE OF GLUCOCORTICOID TREATMENT IN ONCOLOGY INPATIENTS}

Rebecca Benbow. Queen Elizabeth Hospital Birmingham, Edgbaston, Birmingham

\subsection{6/bmjspcare-2018-ASPabstracts.199}

Background Glucocorticoids are widely used for symptom and disease control in patients with cancer. Despite this, there is no recent data on the prevalence of glucocorticoid treatment, or guidance regarding weaning, within this population. This study aimed to determine the point prevalence of glucocorticoid treatment within oncology inpatients at a large tertiary hospital.

Methods On 08.08.2017, the notes of all oncology inpatients in the hospital were reviewed $(n=50)$. Further data was then collected regarding: cancer diagnosis, glucocorticoid indication, weaning plan, and capillary blood glucose (CBG) measurement in the preceding 24 hours.

Results 18 out of 50 (36\%) oncology inpatients were taking glucocorticoids. The underlying cancer diagnoses were skewed towards rarer cancers (sarcomas $n=7$, brain tumours $n=3$, other diagnoses $n=8$ ), reflecting the study's tertiary setting.

The reasons for glucocorticoid treatment included cerebral oedema, queried metastatic spinal cord compression, and immunotherapy related complications.

One patient had a documented short prognosis and was therefore excluded from further analysis. Of the remaining 17 patients,13 (77\%) had documented evidence of a glucocorticoid weaning plan in the previous week. This included 5 patients who were prescribed glucocorticoids as part of an elective inpatient chemotherapy regime.

Only 6 patients out of $17(35 \%)$ had a recorded CBG in the preceding 24 hours; this includes 3 patients with pre-existing diabetes.

Discussion This study, though small, demonstrates the high prevalence of glucocorticoid use within oncology. Weaning of glucocorticoids appears to have been considered in most patients, though not all. Measuring CBGs does not appear to be routine, though it was not possible to determine which patients were at high risk of steroid induced hyperglycaemia. 
This pilot suggests further study into the prevalence and management of glucocorticoids in patients with cancer, across all settings, would be beneficial to establish the need for formal guidelines.

\section{IDENTIFYING INAPPROPRIATE PRESCRIBING IN OLDER PEOPLE WITH DEMENTIA: A SYSTEMATIC REVIEW}

Deborah G Hukins, Una Macleod, Jason W Boland. Wolfson Palliative Care Research Centre, Hull York Medical School, University of Hull

\subsection{6/bmjspcare-2018-ASPabstracts.200}

Background Older people with dementia are at risk of adverse drug-related events associated with the prescribing of potentially inappropriate medications.

Aim To describe how tools designed to identify potentially inappropriate prescribing have been used in studies of older people with dementia and to determine the prevalence of potentially inappropriate prescribing in this cohort.

Methods Systematic review with narrative synthesis designed and conducted according to the recommendations set out in Preferred Reporting Items for Systematic Reviews and MetaAnalyses-Protocol (PRISMA-P) and PRISMA statements. Electronic databases, including MEDLINE, EMBASE, PsychINFO, Cochrane Library, Social Science Citation Index, OpenGrey and Grey Literature Report were searched, along with handsearches, for studies using tools/criteria to identify potentially inappropriate prescribing in older people with dementia. Screening, extraction and quality assessment (Hawker score) were performed in duplicate.

Results A total of 3,626 unique records were identified; 26 studies met the inclusion criteria (which included 26,534 participants, of which $21,285(80 \%)$ had dementia or cognitive impairment). The mean participant age in these studies ranged from 72.5 to 86.8. The Hawker score ranged from 22/36 to $36 / 36$, indicating all studies were fair to good quality. The Beer's criteria was the most commonly used tool (15/26 studies). There were variations in how the tools were applied, with 13 out of 15 studies using the Beer's criteria not using the full tool. Eight studies used more than one tool to identify potentially inappropriate prescribing. The prevalence of potentially inappropriate prescribing ranged from $14 \%$ to $74 \%$ in older people with dementia. The most commonly prescribed potentially inappropriate medications were benzodiazepines and anticholinergics.

Conclusions Variations in tool application may partly explain variations in potentially inappropriate prescribing rates across studies. Recommendations include a more standardised tool usage, ensuring the tools comprehensively identify all potentially inappropriate medications and that the tools are kept up to date.

\section{AN OBSERVATIONAL STUDY OF OPIOID-INDUCED CONSTIPATION (OIC)}

C Leach, A Davies. Royal Surrey County Hospital, Guildford

\subsection{6/bmjspcare-2018-ASPabstracts.201}

Background Opioid-induced constipation (OIC) is common, although there is a lack of consensus on diagnostic criteria, and as a result a lack of consistency around the prevalence (with resultant impact on patient care). This study primarily aims to compare different methods of assessing OIC in a heterogeneous group of patients with cancer.

Methods Data was collected on 100 consecutive patients with cancer that were receiving regular opioid analgesics. Constipation was assessed using a simple question ( 'are you constipated'), the EAPC criteria, the so-called 'Camilleri' criteria (for OIC), the Rome IV criteria (for OIC), and assessment by a specialist palliative care professional. The degree of OIC was assessed using the bowel function index (BFI).

Results The median age was 62 (range 37-86); 46\% were female, 54\% were male. Cancer diagnoses were as follows: $34 \%$ gastrointestinal, $13 \%$ gynaecological, $12 \%$ head and neck, 11\% urological, 11\% breast, 10\% lung, 5\% haematological, $2 \%$ skin, $2 \%$ malignancy of unknown origin.

The prevalence of constipation using the different diagnostic criteria was:

- Simple question - 35\% (with 17\% 'unsure')

- EAPC criteria - 29\% (with 6\% 'unsure')

- Camilleri criteria (for OIC) - 63\% (with 6\% 'unsure')

- Rome IV criteria (for OIC) - 71\%

- Assessment by specialist palliative care professional - $68 \%$ had constipation; of which $44 \%$ had OIC.

Of the patients identified as having OIC from the Rome IV criteria, 30\% thought they were constipated, and the mean BFI was 65. In contrast, the mean BFI for patients that did not meet the Rome IV criteria was 32 .

Conclusions The prevalence of OIC depends on the diagnostic criteria employed. It appears that many patients with OIC do not realise that they are in fact constipated (based on objective criteria).

\section{SEIZURE MANAGEMENT IN PATIENTS UNABLE TO TAKE ORAL MEDICATIONS: A MULTI-CENTRE AUDIT}

Alison Cran, Clare Statham, Harriet Bush, Claire Stark-Toller. University Hospital Southampton NHS Foundation Trust

\subsection{6/bmispcare-2018-ASPabstracts.202}

Background Seizures are common in the palliative care population. For patients who are unable to take oral medication, seizure management can become problematic but there is little consensus on best practice, particularly for patients with longer prognoses.

Aim To examine prescribing of non-oral and 'as required' anticonvulsant medication across multiple palliative settings (community/hospice/acute hospital).

Method A regional multi-centre audit of prescribing practice was conducted from October to December 2017. Inclusion criteria: adult patients requiring anticonvulsant medication but unable to take oral preparations. Practice was assessed against regional Palliative Care guidelines. Data was collected retrospectively via a SmartSurvey proforma.

Results Twenty-six patients across six centres were included. When unable to take oral medications, 25 patients (96\%) commenced a continuous subcutaneous infusion (CSCI). Fourteen patients were prescribed levetiracetam (250 mg-3 g/24 hours), seven were prescribed midazolam (15-30 mg/24 hours), two were prescribed sodium valproate $(600 \mathrm{mg} / 24$ hours) and two were prescribed combination levetiracetam (3 g) and midazolam $(30-40 \mathrm{mg})$. One patient had no regular 